\title{
Phase retrieval algorithm for line-scan dispersive interferometry
}

\author{
Dawei Tang, Prashant Kumar, Feng Gao, Xiangqian Jiang \\ EPSRC Centre for Innovative Manufacturing in Advanced Metrology, University of Huddersfield, \\ Huddersfield HD1 3DH, UK
}

\begin{abstract}
The line-scan dispersive interferometry (LSDI) benefits from single-shot measurement in nature and has potential to perform in-line surface metrology. In this technique, the interference beam produced by the two arms of the interferometer is spatially dispersed by a diffraction grating along the rows (or columns) of the CCD pixels. In which case, a twodimensional spectral interferogram is generated. In this paper, fringe order determination is carried out to retrieve the more accurate phase information along the chromaticity axis of the interferogram and then the height map of the tested profile can be calculated with high resolution. Two standard artefacts have been evaluated using the developed LSDI and the experimental results are compared with that of phase slope method as well as the commercial instrument (Talysurf CCI 3000), which shows that better performance in measurement noise is achieved. Additionally, the measurement repeatability is significantly improved and demonstrated within sub-nanometer range.
\end{abstract}

Keywords: Dispersive interferometry, surface metrology, spectral interferogram, phase retrieval.

\section{INTRODUCTION}

In recent times, significant development in electronics, software and high performance computer has made optical interferometry a popular technique for metrological applications. According to the light source used in the interferometer, interferometric techniques can be generally divided into two main categories, namely monochromatic interferometry (including single wavelength and multi-wavelength interferometry) and white light interferometry. Monochromatic interferometry can achieve surface measurement with low noise and a high resolution of the level of angstrom. Nevertheless, it is limited to the measurement of relatively smooth surfaces due to the well-known $2 \pi$ phase ambiguity problem [1-2]. Line-scan dispersive interferometry (LSDI) [3-4] has been considerable interest due to an advantage of single-shot measurement without $2 \pi$ phase ambiguity and could be potentially applied to in-line surface inspection. By spatially dispersing the interference beam produced by an interferometric objective, a spectral interferogram is obtained with the incoherent superposition of numerous monochromatic interferograms. The phase information is encoded as a function of wavenumber along the chromaticity axis of the camera [5]. Therefore, the depth information of the measured profile can be obtained by interpreting a single two-dimensional frame.

Several analysis techniques have been proposed to analyze the spectral inteferogram, including phase shifting (temporal and spatial) [5], Fourier transform [6], Hilbert transform [7], and convolution [8]. The temporal phase shifting technique is independent on the neighbor pixels and provides accurate results, however, several frames are required to calculate the phase. The spatial phase shifting method only needs single frame to obtain the phase-shifted intensity data. It was however demonstrated to be prone to errors because the intensity variation is not only following the interference equation but also affected by the spectral distribution of light source and spectral response of the camera. A comparison of several single frame methods for phase retrieval was made by Sanjit, et al [9]. In our previous work [10], we applied fast Fourier transform to interpret the fringe pattern in spectral domain, and calculated the phase slope to acquire the absolute height profile. In this paper, firstly, a bench-top LSDI using a 4X Michelson interferometric objective was built. For the fringe analysis, we discuss here the determination of fringe order from the interference signal to improve the measurement resolution, which gives repeatability comparable to phase shifting technique. 


\section{PHASE RETRIEVAL FOR LSDI}

\subsection{Interfe rome try principle}

Interferometry makes use of the interference principle of two beams originating from the same source but travelling on different paths in the interferometric objective, and provides nanoscale vertical resolution for precise surface metrology by analyzing the captured interferogram. As for the LSDI, the spectral intensity recorded at the output of interferometer can be mathematically described as formula [11]

$$
I(z, k)=S(k)\left[I_{R}+I_{M}+2 \sqrt{I_{R} I_{M}} \cos \left(\varphi(z, k)+\varphi_{0}\right)\right]
$$

Where $S(k)$ is the power spectral density of the light source, and $I_{R}, I_{M}$ represent the intensities of the reference arm and measurement arm, respectively. $\varphi(z, k)$ is the phase term related to the optical path difference (OPD) as well as the wavenumber $k$, and $\varphi_{0}$ is the initial phase due to reflection. More specifically, the round-trip OPD equals to $2 z$ and the phase is determined by

$$
\varphi(z, k)=4 \pi k z
$$

\subsection{Phase retrieval}

Interpreting the fringe pattern generated by a white light source is performed to retrieve the phase information and subsequently calculate the height profile of the tested surface. The equation (1) can be written in another form as

$$
I(z, k)=a(k)+b(k)+b^{*}(k)
$$

With

$$
b(k)=\exp [i \varphi(z, k)]
$$

Where $a(k)$ is the background intensity and $b(k)$ is the fringe visibility, and $*$ denotes a complex conjugate. By applying a fast Fourier transform to equation (4), the original spatial signal is described in the frequency domain. Therefore, the unwanted background variation can be removed with a filtration window. Finally, the frequency peak with phase information is performed with inverse fast Fourier transform(IFFT). Taking the natural logarithm of the IFFT formula and then the phase information corresponding to each geometric surface point is obtained by the imaginary part of $\boldsymbol{l n}$ $\exp [i \varphi(z, k)]$.

Because the extracted phase produces a phase modulo $2 \pi$ as a function of wavenumber $k$, the phase unwrapping is required to obtain the continuous phase variation with respect to $k$, which is illustrated in detail by Takeda et. al. in 1982 [12]. According to equation (2), the absolute height value can be determined by the unwrapped phase slope $\mathcal{S}$, as shown in formula

$$
z=\Delta \varphi(z, k) / 4 \pi \Delta k=\mathcal{S} / 4 \pi
$$

\subsection{Fringe order de termination}

The height $z$ obtained by phase slope method is close to the actual value and it can be further improved. This $z$ value is used to estimate the fringe order $\mathcal{F}_{\mathrm{N}}$ of the spectrally resolved interference signal, which is described by formula [13]

$$
\mathcal{F}_{N}(i)=\operatorname{Round}\left(\left(\varphi_{i}-4 \pi k_{i} z\right) / 2 \pi\right)
$$

The function Round ( ) generates the nearest integer value of $\mathcal{F}_{N}$. Therefore, the improved height value using fringe order method has a single wavelength resolution and is expressed as

$$
z_{f}=\left(\varphi_{i}-2 \pi \mathcal{F}_{N}(i)\right) / 4 \pi k_{i}
$$

To demonstrate the enhancement, simulated interference patterns $I_{s}$ with different absolute distances and linear wavenumber across the sampling axis were processed using both phase slope method and fringe order method to make a comparison. Different levels of white noises (none, $50 \mathrm{~dB}$ and $15 \mathrm{~dB}$ ) were added to $I_{S}$ with consideration of the actual 
interference signals. The OPD in this simulation is given between $20 \mu \mathrm{m}$ to $80 \mu \mathrm{m}$ and Figure 1 shows the simulation results. It is found that fringe order technique greatly improves the measurement resolution to nanometer scale, comparing to that of tens of nanometers for phase slope method. It offers stable measurement accuracy even with $15 \mathrm{~dB}$ noise.

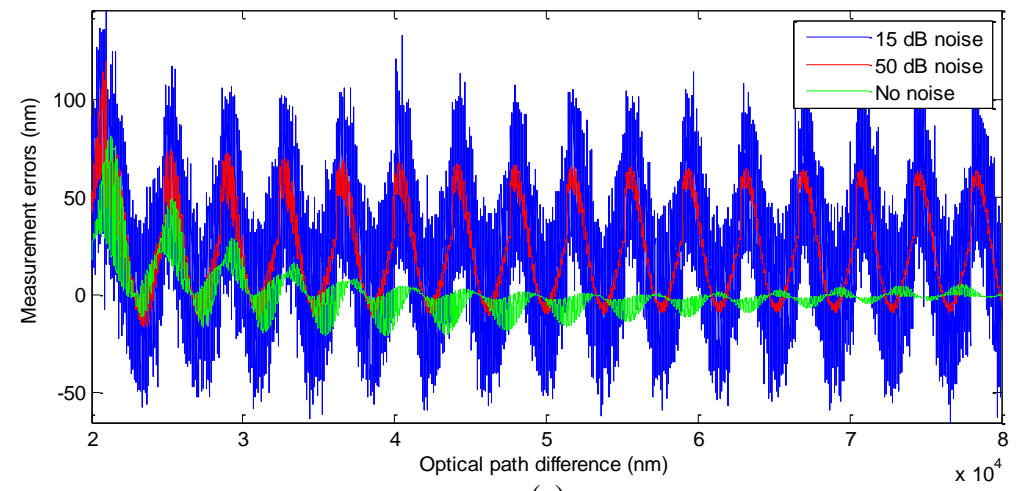

(a)

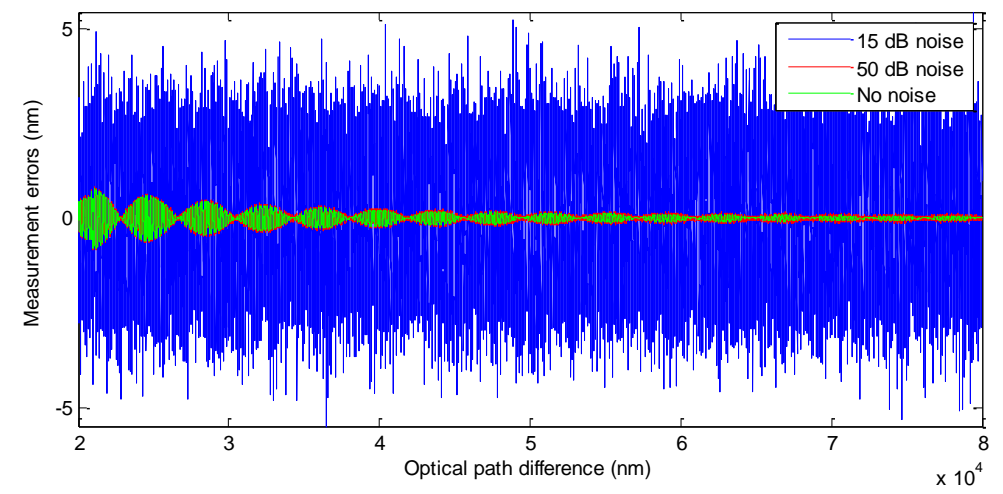

(b)

Figure 1. Simulation results. (a) phase slope method; (b) fringe order method

\section{EXPERIMENTS AND RESULTS}

\subsection{LSDI experimental setup}

The basic configuration of the LSDI systemis illustrated in Figure 2. A halogen bulb with broadband spectrumprovides the white light illumination for the system. A $4 \mathrm{X}$ Michelson interferometric objective is employed to resolve the features on the tested surface. Interference occurs when the two light beams reflected from the reference arm and measurement arm are brought together. Then this interference beam is focused by a spherical tube lens and split into two parts by a beamsplitter. A CCD camera is set at the reflected optical branch to provide real-time images of the tested surface. The other optical branch is brought to a spectrometer to produce spectral interferogram for surface profile measurement. The slit is used to block the light redundant for measurement and is set to be parallel to the columns of camera pixels in the optical arrangement, therefore the dispersion axis is along the rows of the pixels.

System calibrations have been done in terms of wavelength registration and measurement ranges [10]. The result shows that the systemcovers a range of $1.50 \mu^{-1}$ to $1.65 \mu^{-1}$ for wavenumber $k$, corresponding to wavelength span of 605.07 $\mathrm{nm}$ to $657.21 \mathrm{~nm}$. Lateral sampling resolution was calculated as $1.826 \mu \mathrm{m}$ using a microscope slide (R1L3S1P, Thorlabs), and the measurable profile length is obtained as $876.712 \mu \mathrm{m}$. 


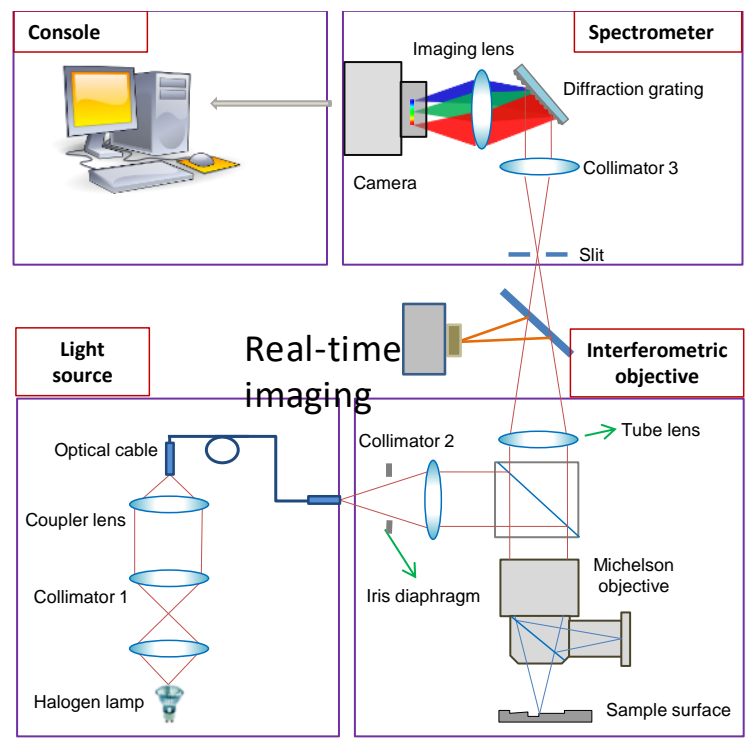

Figure 2. Schmatic diagram of LSDI system

\subsection{Experimental results and discussion}

An optical flat was measured 100 times at the same position and a subtraction technique is adopted for estimating the measurement noise [14]. It is found that the developed LSDI has an average measurement noise of $0.6 \mathrm{~nm}$ with an associated standard deviation (STD) of $0.02 \mathrm{~nm}$ using fringe order method. Whist the phase slope method is applied, the measurement noise is $9.7 \mathrm{~nm}$ with a STD of $0.3 \mathrm{~nm}$. The results are shown in figure 3 as a function of the number of measurements recorded. To make a better visualisation, the mean values have been subtracted from the estimated noises. In conclusion, the performance has been achieved by a factor of fifteen.

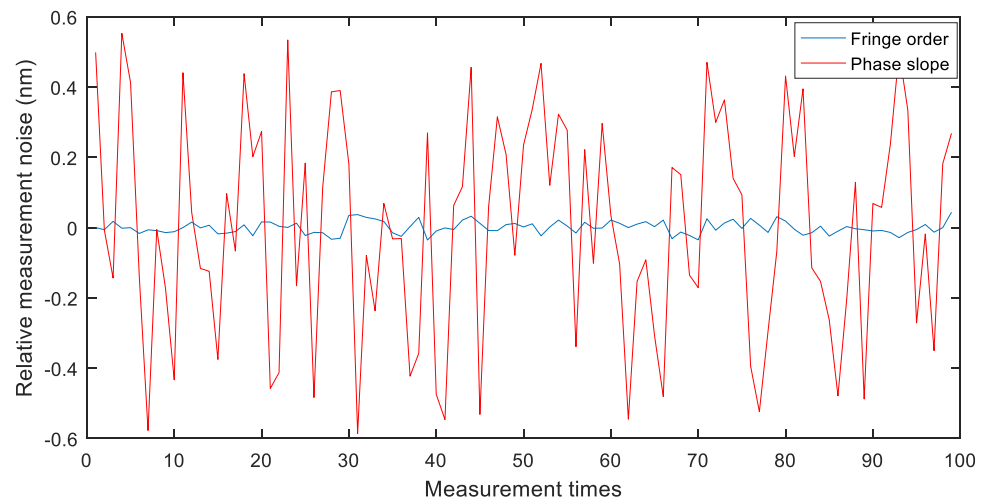

Figure 3. Evaluation of measurement noise (LSDI)

To verify the measurement accuracy and repeatability of LSDI, a standard step artefact (from VLSI, USA) was evaluated. It was calibrated with a nominal height of $178.5 \pm 2.0 \mathrm{~nm}$. Figure 4 are the measurement results using a commercial instrument (Talysurf CCI 3000), which gives a mean height value of $181.9 \mathrm{~nm}$. In Figure 5 the measurement results by LSDI are depicted using two analysis methods for a comparison. The obtained mean height values are $192.7 \mathrm{~nm}$ and $179.2 \mathrm{~nm}$ corresponding to phase slope and fringe order method, respectively. It is apparent that the fringe order result is in good agreement with the nominal value provided the manufacturer. Furthermore, the improvement is clearly noticeable as well when comparing the profiles by two techniques (as well as Talysurf CCI results). The amplitude of the ripples on the measured profile is reduced from the order of tens of nanometers to nanometer scale when the fringe order technique is applied. 
Likewise, by measuring the surface profile on the step artefact 100 times, the measurement repeatability can be assessed and shown in Table 1. The analysis result using fringe order method is depicted in Figure 6 as well with respect to the measurement times. It is found that the measurement repeatability is improved to sub-nanometer range.

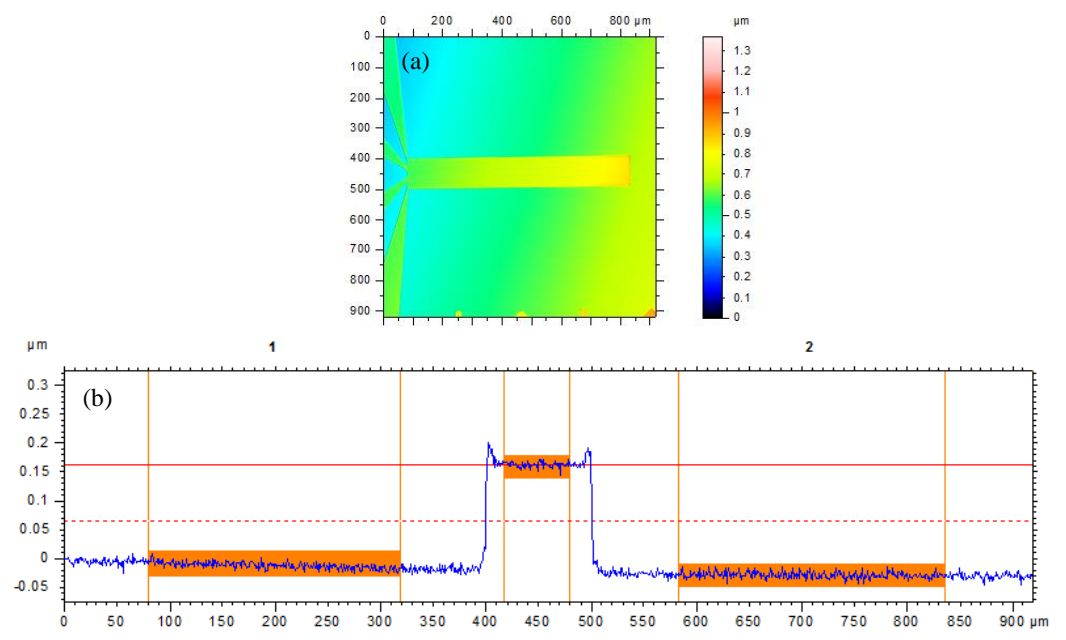

Figure 4. Measurement results using Talysurf CCI 3000. (a) 3D surface map; (b) 2D profile

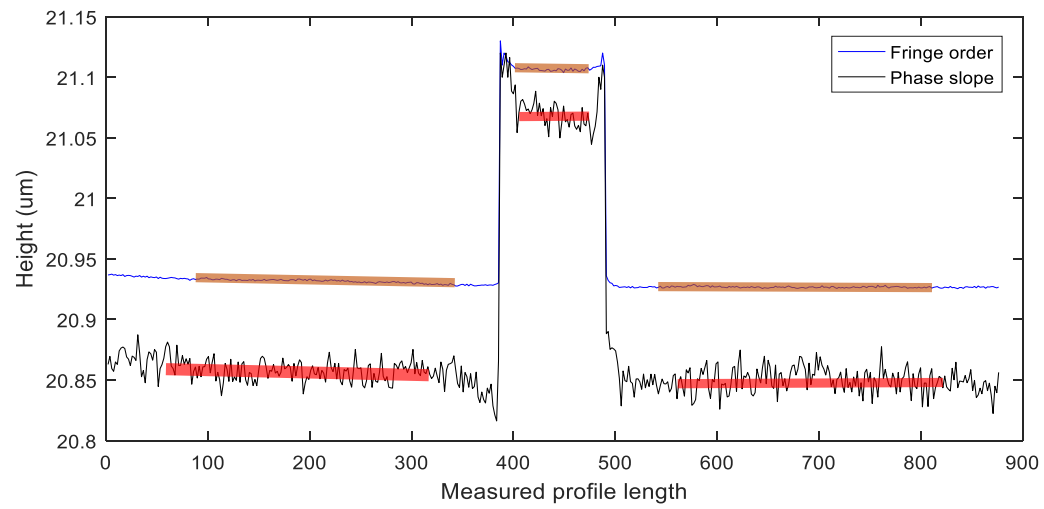

Figure 5. Measurement results of $178.5 \mathrm{~nm}$ step artefact (LSDI)

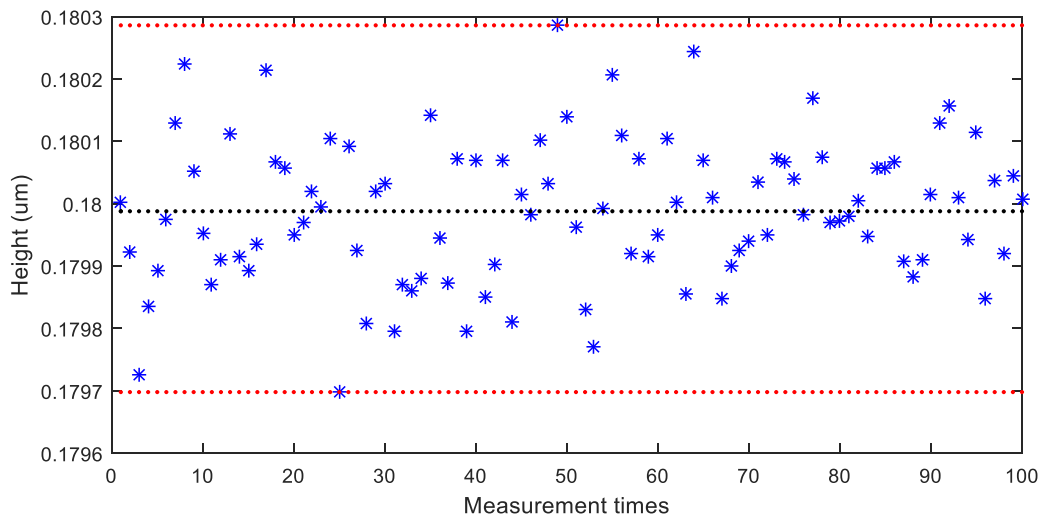

Figure 6. Evaluation of measurement repeatability using fringe order (LSDI). Black broken line is the mean value of the measurements; red broken lines represent one standard deviation from the Mean line. 
Table 1. Comparison of the measurement repeatability

\begin{tabular}{llll}
\hline Methods & Mean height value (nm) & Standard deviation (nm) & Peak-to-valley (nm) \\
\hline Phase slope & 193.9 & 1.9 & 10.8 \\
Fringe order & 180.0 & 0.1 & 0.6 \\
\hline
\end{tabular}

\section{CONCLUSION}

In this paper, implementing the fringe order determination in phase retrieval of the spectral interferogram is reported. Comparisons have been made with the phase slope method to demonstrate the improvement using both theoretical simulation and practical measurements. Two standard artefacts were evaluated by the developed LSDI system as well as a commercial optical instrument. The experiment results show that the algorithm effectively enhances the measurement resolution to a comparable level with phase shifting interferometry. The extended dynamic range also expands the scope of potential applications of the LSDI system in nanoscale surface metrology.

The authors gratefully acknowledge the UK's Engineering and Physical Sciences Research Council (EPSRC) funding of the Future Metrology Hub (Grant Ref: EP/P006930/1), the funding from the Royal Academy of Engineering (RCSRF15161217), the funding with Grant Ref: EP/K018345/1 and the funding of the EPSRC HVMC Fellowship.

\section{REFERENCES}

[1] Malacara, D. ed., Optical shop testing ( $3^{\text {rd }}$ Edition ,Vol. 59), John Wiley \& Sons, Inc., New Jersey (2007).

[2] Creath K., "Step height measurement using two-wavelength phase-shifting interferometry," Applied optics 26, no. 14, 2810-2816, (1987).

[3] Debnath, S. K. and Kothiyal, M. P., "Optical profiler based on spectrally resolved white light interferometry, " Optical Engineering, 44(1), 013606, (2005).

[4] Kumar M., Islam M. N., Terry F. L., Aleksoff C. C. and Davidson D., "High resolution line scan interferometer for solder ball inspection using a visible supercontinuumsource," Optics express 18, no. 21, 22471-22484, (2010).

[5] Debnath, S. K. and Kothiyal, M. P., "Improved optical profiling using the spectral phase in spectrally resolved whitelight interferometry," Applied optics, 45(27), 6965-6972, (2006).

[6] Muhamedsalih, H., Gao, F. and Jiang, X., "Comparison study of algorithms and accuracy in the wavelength scanning interferometry, " Applied optics, 51(36), 8854-8862, (2012).

[7] Debnath, S. K. and Kothiyal, M. P., "Analysis of spectrally resolved white light interferometry by Hilbert transform method, " Proc. SPIE 6292, Vol. 6292, p. 62920P, (2006).

[8] Sainz, C., Calatroni J. and Tribillon G., "Refractometry of liquid samples with spectrally resolved white light interferometry, " Meas. Sci. Technol. 1, 356-361 (1990).

[9] Debnath, S. K., Kothiyal, M. P. and Kim, S. W., "Evaluation of spectral phase in spectrally resolved white-light interferometry: Comparative study of single-frame techniques, " Optics and Lasers in Engineering, 47(11), 1125-1130, (2009).

[10] Tang, D., Gao, F. and Jiang, X., "On-line surface inspection using cylindrical lens-based spectral domain lowcoherence interferometry, " Applied optics, 53(24), 5510-5516, (2014).

[11] You, J. and Joo, K. N., "Minimization of spectral phase errors in spectrally resolved white light interferometry by the iterative least-squared phase-shifting method," Measurement Science and Technology, 23(12), 125203, (2012).

[12] Takeda, M., H. Ina and S. Kobayashi, "Fouriertransform method of fringe-pattern analysis for computer-based topography and interferometry, " J. Opt. Soc. Am. 72, 156-160 (1982).

[13] Upputuri, P. K., Gong, L., Wang, H., Pramanik, M., Nandigana, K. M. and Kothiyal, M. P., "Measurement of large discontinuities using single white light interferogram," Optics express, 22(22), 27373-27380, (2014).

[14] Giusca, C. L. and Leach, R. K., "Calibration of the scales of areal surface topography measuring instruments: part 3. Resolution. Measurement Science and Technology," 24(10), 105010, (2013). 